\title{
Théories et modèles à l'épreuve du terrain : la mobilité comme enjeu de formation
}

\section{Nathalie Auger}

\section{(2) OpenEdition}

12 Journals

\section{Édition électronique}

URL : http://journals.openedition.org/rdlc/7109

DOI : $10.4000 /$ rdlc. 7109

ISSN : 1958-5772

Éditeur

ACEDLE

\section{Référence électronique}

Nathalie Auger, "Théories et modèles à l'épreuve du terrain : la mobilité comme enjeu de formation », Recherches en didactique des langues et des cultures [En ligne], 16-2 | 2019, mis en ligne le 16 novembre 2019, consulté le 20 novembre 2019. URL : http://journals.openedition.org/rdlc/7109; DOI : 10.4000/ rdlc.7109

Ce document a été généré automatiquement le 20 novembre 2019.

\section{c) $(9)$}

Recherches en didactique des langues et des cultures is licensed under a Creative Commons AttributionNonCommercial-NoDerivatives 4.0 International License 


\title{
Théories et modèles à l'épreuve du terrain : la mobilité comme enjeu de formation
}

\author{
Nathalie Auger
}

\section{Oser la mise en œuvre des théories sous la forme d'un modèle}

1 En préambule à la réponse au texte de Marisa Cavalli et Myriam Egli, il me semble important de souligner le courage de la proposition qui s'efforce non seulement de conceptualiser, mais aussi de présenter un modèle qu'elles éprouvent ensuite dans des situations concrètes. Cette approche permet d'affiner le modèle ou les théorisations ayant aidé à le construire (et qui se veulent pluridisciplinaires). Cette méthodologie de réflexion « hélicoïdale » redonne ses lettres de noblesses et toute sa raison d'être à la didactique, point de passage des théories à la pratique et réciproquement, dans une forme de perpétuelle relation entre la didactique descendante et ascendante. En formation comme en recherche, il n'est pas question d'appliquer des théories ou de mettre en œuvre des pratiques coupées des épistémès mais de faire dialoguer ces pôles en se référant, dans une approche maximaliste de la complexité, aux différents publics en mobilité.

2 Le courage du texte de Marisa Cavalli et Myriam Egli est probablement également de considérer les effets sur les apprenants de leur proposition, avec le risque d'être taxées de positivistes (généralement par les didacticiens qui ne formulent pas de propositions). Ce texte a donc le mérite de renouer avec une notion forte de la didactique des langues-cultures (DDLC), celle «d'éducation» (Abdallah-Pretceille \& Porcher, 1998), donc d'accompagnement, forcément subjective et personnalisée selon les apprenants. La question la plus pertinente à se poser en amont est probablement celle des objectifs que l'on poursuit en proposant des descripteurs, des modèles, ou des pratiques de classe. A ce titre, il me semble que les auteurs ont bien clarifié ces 
éléments, gages d'éthique scientifique et d'engagement personnel également indispensables à des formations de qualités.

\section{Le modèle à l'épreuve des enfants migrants en France}

3 J'ai tenté de voir comment le modèle pouvait répondre à un public avec lequel je travaille depuis presque 20 ans : les enfants migrants, afin de comprendre comment il prend en compte les mobilités physiques et symboliques des individus. Le premier point qui a retenu mon attention est celui de la temporalité, la focale sur les relations entre passé, présent et futur dans les dispositifs envisagés. Cet aspect est indispensable car il nous renvoie à notre condition humaine, à notre fonctionnement cognitif qui ne peut vivre que le présent en nous référant sans cesse à nos expériences passées tout en nous projetant dans l'avenir. Si l'on tente donc de transférer les modèles proposés sur d'autres publics que les jeunes en mobilités comme les enfants migrants par exemple (même si dans l'article, visiblement d'autres publics ont, depuis nos discussions, fait l'objet d'attention de la part des auteurs), le texte fait réfléchir sur les potentialités de transferts des propositions théoriques et pratiques. Dans ce contexte de mobilité liée à la migration, la question du passé est primordiale. La difficulté pour ces enfants n'est pas réellement le fait d'arriver, d'être dans ce présent de la classe, mais surtout celui d'être parti. Dans cette configuration, recourir aux biographies langagières (Molinié, 2006 ; Busch, 2006 ; Prasad et Auger, 2015) ou d'autres types de pratiques peuvent être pertinentes mais parfois difficiles. De nombreuses études de terrain, témoignages d'élèves, réfugiés, enseignants expriment leur malaise face à des histoires passées voire à des traumas dont ils ne savent que faire. En formation, le rôle d'enseignant s'en trouve bouleversé. Il sera donc intéressant que la didactique s'entrelace avec la pyschologie/psychatrie (voir les travaux de Moro, 2012) pour proposer des approches du trauma, du passé et l'intégrer dans une approche très benvenistienne (1976) finalement qui rend compte des discours selon un temps (passé-présent-futur) et des personnes en situation. Par ailleurs, concernant les publics migrants, les études (Auger, 2010) montrent le plus souvent un fort investissement dans le présent et une projection dans l'avenir très marquée. Nul doute que les enseignants en formation auront besoin de ces connaissances.

4 Un autre élément très pertinent du texte de Marisa Cavalli et Myriam Egli est relatif à la question de la mobilité dans sa relation à l'altérité, à ce qu'elles appellent de « nouvelles communautés ». Les pôles, les tensions manifestées dans le modèle proposé, si on les transpose aux publics migrants seraient également à corréler avec la notion de vitesse. Pour ces publics, la vitesse de rencontre avec les nouvelles communautés est extrêmement rapide et sans préparation le plus souvent. Cette notion de vitesse est probablement un paramètre très intéressant à intégrer au schéma proposé. Vitesse et distance sont deux principes qui peuvent permettre d'interroger, en recherche comme en salle de classe, des approches bien variables entre les langues, les communautés. Autant d'impensées actuelles de la DDLC. Car si ces études sur la distance linguistique, les temporalités d'apprentissage existe dans la formation des enseignants au Canada, il n'y a pas de balisage en formation d'enseignants sur la notion de distance entre communautés. D'aucuns pourraient dire que l'interculturel joue ce rôle mais cette approche est davantage de l'ordre de l'individuel et non du groupe. Marisa Cavalli et Myriam Egli ont intégré cette notion de groupe avec raison, car les apprenants les 
éprouvent en situation mais ce groupe est peu problématisé en DDLC. Par ailleurs, cette notion de communauté, repris de "communauté de pratique ", que l'on retrouve aussi dans les études récentes sur les usages du numérique ou en Amérique du Nord en didactique, si on l'applique encore aux publics migrants (public qui fait office de fil conducteur pour mettre à l'épreuve le modèle proposé), le terme ne peut esquiver sa familiarité avec "communautarisme». Les travaux de Fabrice Dhume (2016) sur le communautarisme. Enquête sur une chimère du nationalisme français, montrent à quel point ce terme existe en France et n'est pas réellement traduisible dans d'autres langues, ou l'idée de groupes divers constitue le modèle politique du pays comme en Amérique du Nord, contrairement à la France. La question va donc se poser de garder ou traduire cette idée de « communautés » en formation pour les enseignants de langue afin de ne pas générer des stéréotypes contre-productifs pour les pratiques de classe.

Corrélée aux questions de communautés, la notion d'adaptation culturelle et linguistique qui est proposée par de Marisa Cavalli et Myriam Egli est probablement à prendre avec précaution en formation. Si on se réfère épistémologiquement, par exemple, à Piaget (1946) et aux principes d'accommodation et d'assimilation, cette notion n'a rien de répréhensible. Elle gène davantage dans la façon dont elle circule dans les imaginaires collectifs en laissant supposer une transformation unilatérale. La notion de pertinence par rapport à des normes dans un contexte donné permet peutêtre d'échapper à l'idée de compétences " non convenables, inadaptées » que pourrait suggérer « adaptation ».

6 Sur la notion de médiation dans le texte de de Marisa Cavalli et Myriam Egli, l'idée d'une possible auto-médiation sous la forme d'une introspection (journaux réflexifs, Porte-folio Européen des Langues, etc.) est intéressante, à condition, encore une fois, de proposer l'accompagnement nécessaire à des publics qui peuvent être vulnérables. Cela nous ramène aussi, dans une certaine mesure, aux travaux sur la violence verbale à l'école (Auger \& Romain, 2015) ou plus largement dans l'espace public (Fracchiolla, Moïse et al., 2014) car, la relation à l'altérité peut comporter une dimension de malaise, voire de violence (du moins symbolique) et les mécanismes compris pour décrire les relations iréniques complètent les propositions de de Marisa Cavalli et Myriam Egli. En effet, le contexte est le premier paramètre à prendre en ligne de compte dans une situation potentiellement conflictuelle (par exemple, salle peu adaptée aux enfants accueillis : dans une bibliothèque, un coin de classe etc. qui indique une forme de marginalisation des élèves) ou aux individus (mal-être, liés au passé par exemple) et peut donc générer de la violence à terme. Il est intéressant de travailler avec ce contexte et les personnes pour leur offrir de meilleures conditions et empêcher la montée en tension de la violence verbale pour ainsi favoriser la relation à l'altérité qu'il s'agisse de personnes ou d'objets d'apprentissage. Si ces éléments ne sont pas pris en compte, on risque d'entrer dans un engrenage de relations agonales. La médiation est donc presque trop tardive à ce stade même si elle peut encore exister (être le tiers qui reformule les propos de l'un ou de l'autre pour trouver une résolution au conflit, différer la discussion pour abaisser l'émotion etc.). Il est donc plus fructueux de travailler en amont. Finalement, les enseignants sont encore assez peu outillés aux approches de l'analyse du discours et des interactions qui sont pourtant majeures pour être en position de médiateur efficace (utilisation des adoucisseurs, repérage des durcisseurs ; Auger, $2015: 22-40$ ). 


\section{Vers une formation épistémologique pour comprendre l'articulation théorie-modèle}

7 Au-delà des outils ou des concepts très intéressants qui sont proposés dans le texte de Marisa Cavalli et Myriam Egli, le plus important est probablement le processus induit par les dispositifs. Car n'importe quel outil ou approche peut être contreproductif si un processus d'expérimentation n'est pas mis en branle par les conditions de travail. Les outils ont donc le mérite d'exister, ils sont rassurants pour les enseignants mais si des tâches-problèmes qui induisent des processus ne sont pas proposées, la médiation ou l'appropriation de la langue ne peut aboutir. Finalement, les sous-bassement théoriques (socio-constructivisme) sont certainement primordiaux dans la formation des enseignants comme Marisa Cavalli et Myriam Egli l'affirment et telles qu'elles les ont replacés à leurs justes places dans leurs travaux.

Finalement, l'épistémologie est primordiale en formation, tout autant que les modalités de mises en œuvre de ces concepts sous forme de pratiques en salle de classe en prenant en compte les mobilités à la fois physiques et symboliques des apprenants.

\section{BIBLIOGRAPHIE}

Abdallah-Pretceille, M., \& Porcher, L. (1998). Ethique de la diversité et éducation. Paris : PUF.

Auger, N. (2010). Elèves Nouvellement Arrivés en France. Réalités et perspectives en classe. Paris : Editions des Archives Contemporaines.

Auger, N., \& Romain, C. (Eds.). (2015). Violence verbale et Ecole. Paris : L'Harmattan.

Auger, N. (2015). «Violence verbale au travers des classes ZEP : enjeux des langues et des variations ». In Auger, \& C. Romain (Eds.), Violence verbale et Ecole. Paris : L'Harmattan. pp. 22-40.

Benveniste, E. (1976). Problèmes de linguistique générale. Paris : Gallimard.

Busch, B. (2006). « Language Biographies - approaches to multilingualism in education and linguistic research ». In B. Busch, A. Jardine, \& A. Tjoutuku (Eds.). Language biographies for multilingual learning. Cape Town : PREAESA occasional Papers. No. 24, 5-17.

Dhume, F. (2016). Communautarisme. Enquête sur une chimère du nationalisme français. Paris : Éditions Demopolis.

Fracchiolla, B., Moïse, C., Romain, C., \& Auger N. (2014). Violences verbales, analyses, enjeux et perspectives. Rennes : PUR.

Molinié, M. (dir.) (2006). « Biographie langagière et apprentissage plurilingue », Recherche et Application Le Français dans le Monde, $\mathrm{n}^{\circ} 39$.

Moro, M-R. (2012). Les enfants de l'immigration, une chance pour l'école. Bayard éditions : Montrouge.

Piaget, J. (1946). La Formation du symbole chez l'enfant. Genève : Delachaux \& Niestlé. 
Prasad, G., \& Auger, N. (2015). « Mais est-ce que ça existe une personne monolingue?

Plurilinguisme des élèves au Canada et en France, pratiques artistiques et langagières et apprentissage du français ». Carnets d'Ateliers de Sociolinguistique. Université d'Amiens, 20-32.

\section{RÉSUMÉS}

Le texte de Marisa Cavalli et Myriam Egli suscite une réflexion concernant la relation à tisser entre les théories socio-constructiviste et le dispositif proposé. Mis à l'épreuve d'un public comme les enfants migrants en France, cet article questionne les dimensions potentiellement traumatiques de la mobilité de ces publics, les notions de vitesse et de distance relatives à l'inclusion, les sentiments d'appartenances et de communautés qui sont des sujets d'une brulante actualité.

Marisa Cavalli and Myriam Egli's paper gives rise to a reflection on the relationship between socio-constructivist theories and the programme they have designed. The aim of our text is to question this relation regarding migrant children pupils in France specifically. This article questions the potentially traumatic dimensions of the mobility of these students, the notions of speed and distance towards inclusion, and feelings of belonging to communities. These topics are of the upmost importance in the context of today's mobility.

\section{INDEX}

Mots-clés : théories, dispositifs, enfants migrants, mobilité

Keywords : theories, programms, migrant pupils, mobility

\section{AUTEUR}

\section{NATHALIE AUGER}

\section{UMR CNRS Praxiling}

Université Paul-Valéry Montpellier 3

Responsable d'une équipe CNRS sur l'appropriation des langues et du langage, Nathalie Auger mène une réflexion sur ce que l'enseignement du français « langue maternelle » peut signifier dans la France et l'Europe plurilingue / multiculturelle du XXIe siècle (projets de recherches avec les élèves migrant-Comparons nos langues, Maledive, Conbat+, gitans-Gypsylang et rom-Romtels, partenariats avec le Conseil de l'Europe et la Commission européenne). Elle a comparé ses études à l'enseignement du français au Canada (enfants immigrés, immersion en anglais et minorité francophone).

http://www.praxiling.fr/auger-nathalie.html nathalie.auger[at]univ-montp3.fr 